\title{
CODE STEMI Improves Clinical Outcomes and Cost Efficiency in ST Elevation Myocardial Infarction Patients at Dr Cipto Mangunkusumo General Hospital
}

Eka Ginanjar ${ }^{1}$, Amal C Sjaaf ${ }^{2}$, Idrus Alwi ${ }^{1}$, Wahyu Sulistyadi ${ }^{2}$, Ede Suryadarmawan ${ }^{2}$, Adik Wibowo $^{2}$, and Lies Dina Liastuti ${ }^{1}$

${ }^{1}$ Rumah Sakit Umum Pusat Nasional Dr Cipto Mangunkusumo

${ }^{2}$ University of Indonesia Faculty of Public Health

April 28, 2020

\begin{abstract}
Background : One of the highest causes of cardiac mortality is ST Elevation Myocardial Infarction (STEMI). Delay in the management of STEMI patients resulted high mortality, morbidity, and economic burden. CODE STEMI is a protocol to reduce door to balloon time and improve patient's quality care and clinical outcome. Objectives : To determine the effect of the implementation of CODE STEMI on clinical outcome and cost efficiency of a quality care of STEMI patients at Dr. Cipto Mangunkusumo General Hospital. Method: This is a retrospective cohort study that reviewed 207 medical records of STEMI patients who underwent primary percutaneous coronary intervention (PPCI) in 2015-2018. The patients were divided into two groups. The first group was treated prior to establishing the CODE STEMI and the other group was treated with CODE STEMI protocol. The primary outcome was to measure in-hospital mortality and morbidity and total hospital cost between both groups. Secondary end-points included length of stay (LOS) and Cost Efficient Analysis. Data analysis was done using Mann Whitney and Chi square test. Results: There were 72 and 135 patients in Pre-CODE STEMI and CODE STEMI groups respectively. In CODE STEMI group, median D2BT was significantly reduced by 130 min $(\mathrm{P}<0.001)$, median LOS was reduced by 1 day $(\mathrm{P}=0.009)$ and there was cost reduction by approximately USD 1,000. There were declining trend of in-hospital mortality rates ( $8.3 \%$ vs $4.4 \%)$ and MACE at 30 days (48.61\% vs $37.78 \%$ ). Conclusion: Implementation of CODE STEMI can improve clinical outcome, reduce length of stay and cost efficiency of STEMI patients in general hospitals.
\end{abstract}

CODE STEMI Improves Clinical Outcomes and Cost Efficiency in ST Elevation Myocardial Infarction Patients at Dr Cipto Mangunkusumo General Hospital

"An Optimal Program for Myocardial Infarction Patients"

Eka Ginanjar $^{1^{*}}$, Amal C Sjaaf ${ }^{2}$, Idrus Alwi ${ }^{1}$, Wahyu Sulistyadi ${ }^{2}$, Ede Suryadarmawan ${ }^{2}$, Adik Wibowo $^{2}$, Lies Dina Liastuti ${ }^{3}$

${ }^{1}$ Departement of Internal Medicine, Faculty of Medicine University of Indonesia-Dr. Cipto Mangunkusumo Hospital, Jakarta, Indonesia

${ }^{2}$ Faculty of Public Health, University of Indonesia, Depok, West Java, Indonesia

${ }^{3}$ Dr. Cipto Mangunkusumo Hospital, Jakarta, Indonesia

*Corresponding Author : Eka Ginanjar, Division of Cardiology, Department of Internal Medicine, Faculty of Medicine Universtas Indonesia - Dr. Cipto Mangunkusumo General Hospital. Jl. Diponegoro 71, Jakarta 10430, Indonesia, Email : ekginanjar@gmail.com, 
Phone : +62 811868093

\section{Acknowledgments}

We would like to acknowledge Mrs. Utami Susilowati (Biostatistician) for advice regarding statistical analyses. We would also like to thank dr. Valerie Hirsy for their assistance in writing the manuscript, and dr. Ika Prasetya Wijaya as department chairperson who provided general support for this study.

Hosted file

MANUSCRIPT CODE STEMI.docx available at https://authorea.com/users/311684/articles/446345-codestemi-improves-clinical-outcomes-and-cost-efficiency-in-st-elevation-myocardial-infarctionpatients-at-dr-cipto-mangunkusumo-general-hospital

\section{Patient Arrival}

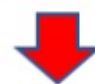

ED Triage

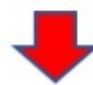

\section{Assessment by ED physician:} Anamnesis, physical exam, ECG

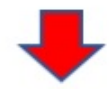

\section{Contact cardiologist consultant}

Confirm STEMI diagnosis to activate CODE STEMI

Alert interventional cardiologist, cardiac catheterisation

laboratory team, clerical and administrative staff

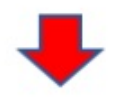

\section{Patient transfer to catheterization laboratory}

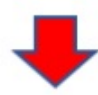

\section{Primary $\mathrm{PCl}$ preparation}




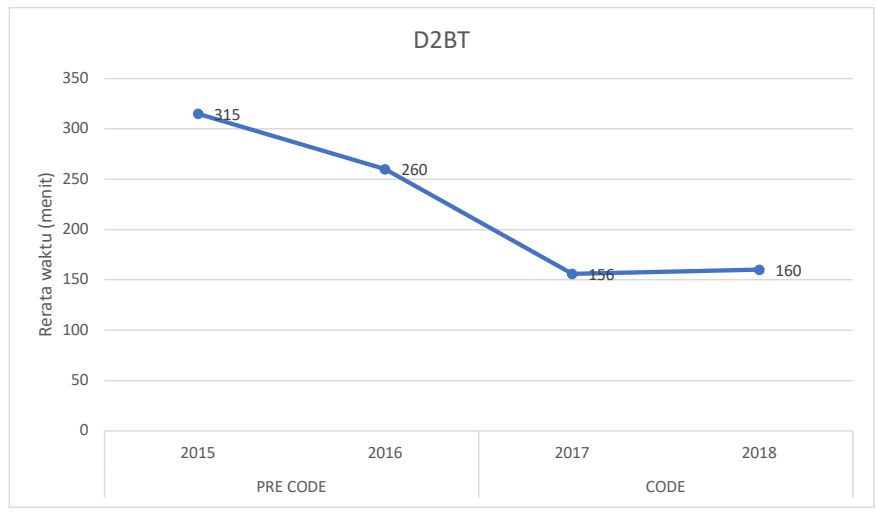

Figure 2. Declining trend of Door to balloon time in STEMI patients by year

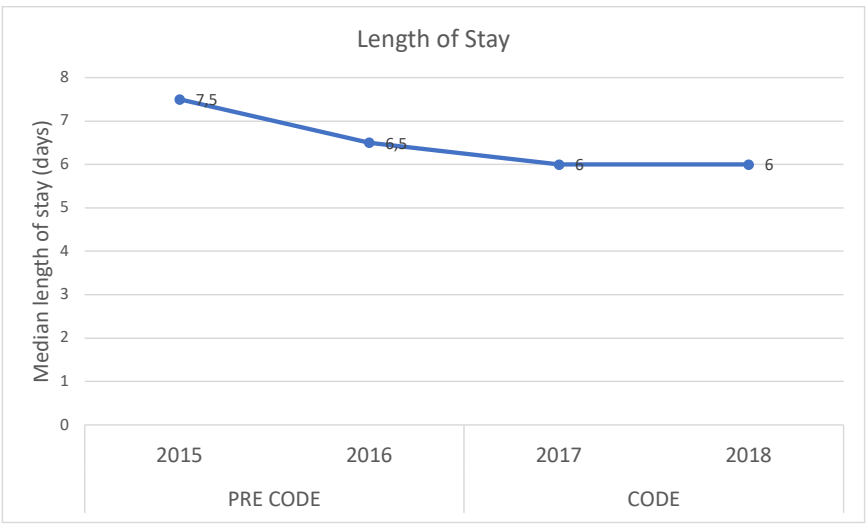

Figure 3. Declining trend of length of stay in STEMI patients by year 